\title{
Fluctuating cognitive abnormalities and cerebral glucose metabolism in neuropsychiatric systemic lupus erythematosus
}

\author{
Ramona M Carbotte, Susan D Denburg, Judah A Denburg, Claude Nahmias, \\ E Steven Garnett
}

\begin{abstract}
Brain imaging techniques such as MRI and PET have the potential for identifying central nervous system involvement in SLE. They may also help elucidate the mechanisms giving rise to the widely dlverging manifestations of CNS involvement in SLE. This report documents an intensive longitudinal study of three women with neuropsychiatric SLE. PET and neuropsychological evaluation were both used to examine the co-occurrence of behavioural/cognitive deficits with alterations in regional brain glucose metabolism. In all three patients, FDG uptake indicated abnormalities which were not identified on CT scan, but corresponded well with localisable cognitive deficits. Changes in each patient's cognitive profile on reassessment paralleled changes on PET. These findings support the suggestion that cognitive deficits in SLE patients reflect primary CNS involvement.
\end{abstract}

(F Neurol Neurosurg Psychiatry 1992;55:1054-1059)

CNS involvement occurs in as many as $75 \%$ of patients with systemic lupus erythematosus (SLE) and is shown in diverse neurological and psychiatric symptoms. ${ }^{12}$ Although the pathogenesis of this condition remains obscure, several mechanisms have been proposed to explain it. They include vasculopathy mediated by immune complexes, thromboembolic disease, and direct antibody-mediated neuronal dysfunction. $^{3-6}$

The prevalence of impaired cognitive function is also high in SLE patients, irrespective of clinically diagnosed CNS involvement at the time of testing. ${ }^{7}$ We have found that two thirds of a large unselected sample of SLE patients showed significant cognitive impairment; such impairment was also present in $40 \%$ of the patients who had never had neuropsychiatric (NP) symptoms and in over $85 \%$ of the patients whose CNS involvement was judged to have been clinically resolved for at least a month. We found no significant association between cognitive impairment and emotional distress, ${ }^{78}$ systemic involvement as indexed by the lupus activity criteria count, ${ }^{9}$ or steroid treatment at the time of testing. ${ }^{78}$ These deficits in cognitive functioning in patients without other concurrent CNS symptoms are thus consistent with subclinical CNS involvement as has been documented in other medical conditions. ${ }^{1011}$

Confirming the presence of CNS abnormalities in NP-SLE can be very problematic even when cognitive testing, history and symptoms suggest CNS involvement. Electrophysiological, radiological and CSF analyses are frequently used but their sensitivity is limited. ${ }^{12}$ MRI appears to be much more sensitive because it has been able to identify focal lesions in NP-SLE patients with clinical evidence of recent strokes or seizures, in whom contrast enhanced CT scans showed no abnormalities. $^{1314}$

Brain images based on metabolic function may prove to be even more sensitive than MRI in documenting the location and extent of CNS involvement in NP-SLE patients. Studies of glucose metabolism, using PET, have identified fluctuating alterations in regional cerebral metabolism in NP-SLE patients, even when no structural lesions were evident on MRI. ${ }^{15-17} \mathrm{~A}$ study of the regional distribution of cerebral oxygen consumption and blood flow, using ${ }^{15} \mathrm{O}$ with a gamma camera, identified abnormalities in 14 of 15 SLE patients with suspected CNS involvement and in 10 of 12 patients in whom CNS disease was not clinically apparent. ${ }^{18}$ Awada et $\mathrm{al}^{19}$ using the $\mathrm{Xe}^{133}$ technique, found greater than normal heterogeneity in the pattern of regional cerebral blood flow ( $\mathrm{rCBF}$ ) in patients without current or previous clinically identified neurological disorder, and Kushner et $a l^{20}$ have reported lower global CBF in periods of exacerbation of CNS involvement, with normal values in time of remission.

None of the radiological or metabolic studies of CNS function in SLE patients with suspected or documented CNS involvement has related abnormalities in brain function to changes in cognitive functioning. Such studies could establish the behavioural correlates of identified lesions in individual patients as well as validate inferences or hypotheses regarding the presence and neural substrates of impairment made solely on the basis of neuropsychological assessment. In turn this would lend strong support for the use of neuropsychological assessment as a diagnostic procedure to identify and characterise CNS involvement in SLE, particularly when PET or MRI are not available. Sequential neuropsychological assessment could also be the method of choice for monitoring the progress of cerebral SLE.

In this paper we present the results of serial cognitive testing and PET in three women with SLE. In each of the three patients, the cognitive findings were interpreted independently of the PET studies, to establish the extent to which the cognitive results indicated changes in CNS function not identified clinically. 


\section{Patients and methods}

Three right handed women satisfying ARA criteria $^{21}$ for SLE gave informed consent to serial evaluation with PET and neuropsychological testing. All three patients had a documented CVA as well as positive ANA, DNA, and lupus anticoagulant as measured by a series of bioassays, including dilute PTT and $\mathrm{Xa}$ inhibitor studies. Cases 1 and 2 had had extensive cognitive testing before their CVA.

Case 1 SLE was first diagnosed at the age of 13. Neuropsychological testing was initially done at the age of 16 to evaluate the neurocognitive sequelae of seizures and generalised encephalopathy with coma from which she had recovered. The first PET study was done when the patient was 18 years old, one month after the onset of left sided hemiparesis, right sided amaurosis fugax, and persistent headache. The clinical picture at the time of testing included arthritis, skin rash, membranoproliferative glomerulonephritis and mild renal insufficiency. Her laboratory results showed mild leucopenia, lupus anticoagulant, normal $\mathrm{C} 3$ and $\mathrm{C} 4$, and $38 \%$ DNA binding. Her CT scan showed mild dilation of the sulci and ventricles, bilateral basal ganglia calcification, and a low density area deep to the right insula probably involving the putamen and external capsular region. She was on $50 \mathrm{mg}$ prednisone, alternate days (which had been increased from $5 \mathrm{mg}$, alternate days, at the time of her stroke). A second PET study was done seven months later, at which time the left-sided hemiparesis had resolved, and she no longer complained of headache or amaurosis. The prednisone dose had been tapered to $5 \mathrm{mg}$ on alternate days.

Case 2 SLE was first diagnosed at the age of 29. The patient had a history of recurrent miscarriages, deep vein thrombosis, polyarthritis, nephritis, livedo reticularis, hypertension, Raynaud's phenomenon, and a two year history of grand mal seizures. At that time, clear EEG abnormalities were not present. Serology was characterised by positive DNA binding, the presence of a lupus anticoagulant, and high titred anti-neuronal antibody, with specificity for a new $97 \mathrm{kd}$ antigen. ${ }^{22}$ At the age of 37, one year before her first PET scan, an EEG showed mild and diffuse slowing of dominant frequencies, with no lateralising, focal or paroxysmal features, and a CT scan showed generalised widening of the sulci and moderate ventricular dilation. Anticonvulsant medications were dilantin $325 \mathrm{mg}$ and valproic acid $2500 \mathrm{mg}$ daily.

The first PET scan was carried out two months after she developed right sided hemiparesis and dysphasia. Her CT scan at this time showed a small left parietal infarct in addition to atrophy. Prednisone had been increased from $25 \mathrm{mg}$ per day to $75 \mathrm{mg}$ after the infarct. A second PET scan was carried out ten months later, at which time the hemiparesis and speech difficulties had resolved. She was taking $25 \mathrm{mg}$ and $5 \mathrm{mg}$ prednisone on alternate days, in addition to $325 \mathrm{mg}$ dilantin and $1500 \mathrm{mg}$ valproic acid.

Case 3 SLE was diagnosed at the age of 31. The patient had a history of rash, arthritis, and mesenteric vein thrombosis. Subsequently, she had recurrent seizures (grand and petit mal). At the age of 34 , she developed symptoms suggesting a left hemisphere stroke, with subsequent "slowed mentation". The first PET scan was carried out at the age of 39. CT scan at this time showed moderate dilation of the ventricles, sulcal widening, and an "old" left parietal infarct. Her EEG showed right midtemporal focal spikes; anticonvulsive treatment was $400 \mathrm{mg}$ dilantin. Serological findings included positive DNA binding and lupus anticoagulant. Prednisone dose had been recently increased to $30 \mathrm{mg} /$ day. A second PET scan was carried out eight months later when the prednisone had been tapered to $15 \mathrm{mg}$ and $17.5 \mathrm{mg}$ on alternate days, but $120 \mathrm{mg}$ phenobarbital had been added to the anticonvulsant treatment.

\section{Neuropsychological Assessment}

Full details of our neuropsychological testing and interpretative procedures have been presented elsewhere. ${ }^{78}$ Our tests have been chosen to assess a wide range of functions; our interpretive procedures allow us both to classify individual patients as cognitively intact or impaired, and also to generate a detailed cognitive profile of relative strengths and deficits for individual patients, on the basis of which we infer areas of probable CNS dysfunction.

The complete neuropsychological assessment included the following tests which are described in detail in Lezak: ${ }^{23}$ Wechsler Adult Intelligence Scale (Information, Comprehension, Similarities, Digit Span, Digit Symbol, Picture Completion, Block Design); Wechsler Memory Scale with delayed recall; Rey Auditory-Verbal Learning; Corsi Block Span; Rey Osterrieth Complex Figure Drawing with delayed recall; Verbal Fluency tests (Animal Naming and Controlled Word Association); Design Fluency; Stroop Colour-Word Interference; Trailmaking; Finger Tapping. Data from all these tests were available from the first assessment but not from some of the subsequent testing sessions for the patients described in this report. In all cases, neuropsychological testing was done on the same day as the PET scanning.

The analysis of the test protocols involves the following data transformations. Each of the test scores is expressed as a standard or z-score. A z-score is simply the extent to which the patient's score deviates from the mean of the control or normative group, divided by the standard deviation of the normative group. After this transformation, scores from different tests can be combined, or directly compared, since they now have a common unit of measurement. Younger and older normative groups for these transformations were obtained by dividing our total normal control group $(\mathbf{N}=35)$ at the median age. The $z$-scores for each individual are subgrouped into a number of different non-overlapping summary scores. To arrive at a decision regarding cognitive status, the mean z-score on each summary variable is compared with an estimate of 
premorbid level of functioning (also expressed as a $\mathrm{z}$-score). Any difference greater than 2 units (standard deviations) between the estimated premorbid level and mean summary score is taken to reflect significant impairment. Any protocol containing more than two such impaired summary scores is interpreted as reflecting significant cognitive impairment.

\section{Positron emission tomography}

Regional dístribution of cerebral glucose metabolism was measured by PET using $\left[{ }^{18} \mathrm{~F}\right]$ 2-fluoro-2-deoxy-D-glucose ( ${ }^{18} \mathrm{~F}$ FDG). The tomograph had a spatial resolution of $8 \mathrm{~mm}$ in the plane and $16 \mathrm{~mm}$ in the axial direction. ${ }^{24}$ Typically, 16 or more slices of the brain were obtained sequentially from the level of hippocampal gyri to the level of the cortical vertex. During the PET procedure, subjects reclined

Figure 1 Distribution of cortical glucose metabolism (umol/100g/min) in the left (left panels) and right hemispheres (right panels). The intercommissural (Ac-Pc) line ${ }^{27}$ is shown on each image: $A$ ) Case 1 (top): distribution of cortical glucose metabolism at one month (upper panel) and eight months (lower panel) after an infarct in the right insula; B) Case 2 (centre): two months (upper panel) and twelve months (lower panel) after a left parietal infarct; C) Case 3 (bottom): before and after changes in steroid treatment.
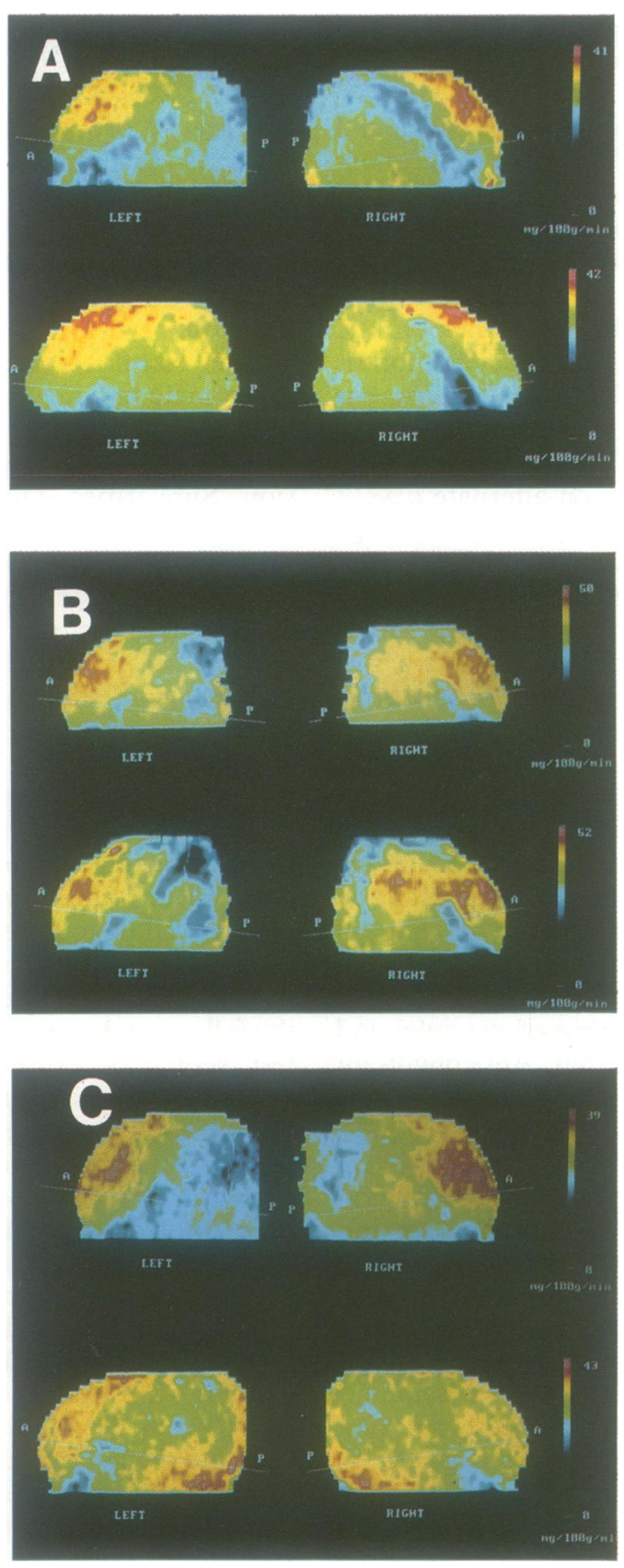

on a comfortable stretcher in a quiet, well lit room. Before the PET study, an indwelling IV catheter for blood sampling was inserted. After a short interval during which the patient became used to the room, $6 \mathrm{mCi}$ FDG was injected intravenously. During the interval between the injection of FDG and the tomographic measurement, subjects were asked to keep their eyes closed and to refrain from talking. Forty five minutes after the injection of FDG, the cerebral distribution of ${ }^{18} \mathrm{~F}$ was measured in the tomograph. Regional cerebral glucose metabolism, CMRglu, was calculated. ${ }^{25}$ Each image was reconstructed on a $128 \times 128$ pixel grid and each pixel was $2 \mathrm{~mm}$ wide. The images were reconstructed using a Shepp-Logan filter and a calculated attenuation correction. The transverse PET sections were analysed ${ }^{26}$ and displayed as paired twodimensional projections of the convexities of the left and right hemispheres (fig 1 , described below).

\section{Results}

Case 1

The first PET scan (fig la) showed a reduction of glucose metabolism along the whole length of the right central sulcus, extending posteriorly into the superior parietal lobule. Similar but rather slight reductions were seen on the left. The second PET scan, carried out seven months later, showed a marked improvement in glucose consumption in the right parietal region and in the upper half of the region of the central sulcus. Inferiorly, the pericentral reduction of glucose consumption persisted. No abnormalities were evident on the left.

There was no evidence of clinically active CNS involvement at the time of baseline testing two years before her CVA; she had also been designated as cognitively unimpaired overall, although she did show some specific verbal memory deficits. Figure 2 is a schematic presentation of changes from this cognitive baseline in a number of summary cognitive measures obtained when she was tested at the time of each PET scanning. Her performance at the time of the first PET study indicated a fairly generalised decline in tests of visualspatial functioning and cognitive flexibility, in spite of improvement in several areas of verbal functioning. This pattern was interpreted as being compatible with widespread right hemisphere involvement. Decreased psychomotor speed and cognitive flexibility were compatible with having a recent CNS event, but nonspecific as to the nature or lateralisation of the event. Decreased verbal productivity suggested frontal involvement; this productivity deficit is most likely to be seen in patients with lesions on the left, but does also occur occasionally with right-sided lesions. Her improvement over baseline performance in verbal memory and reasoning at the time of the first scan indicated a resolution of deficits related to previous CNS events. When reassessed seven months later, she showed a substantial improvement in previously depressed scores, and no significant residual deficits. 


\section{CASE 1}

COGNITIVE VARIABLES

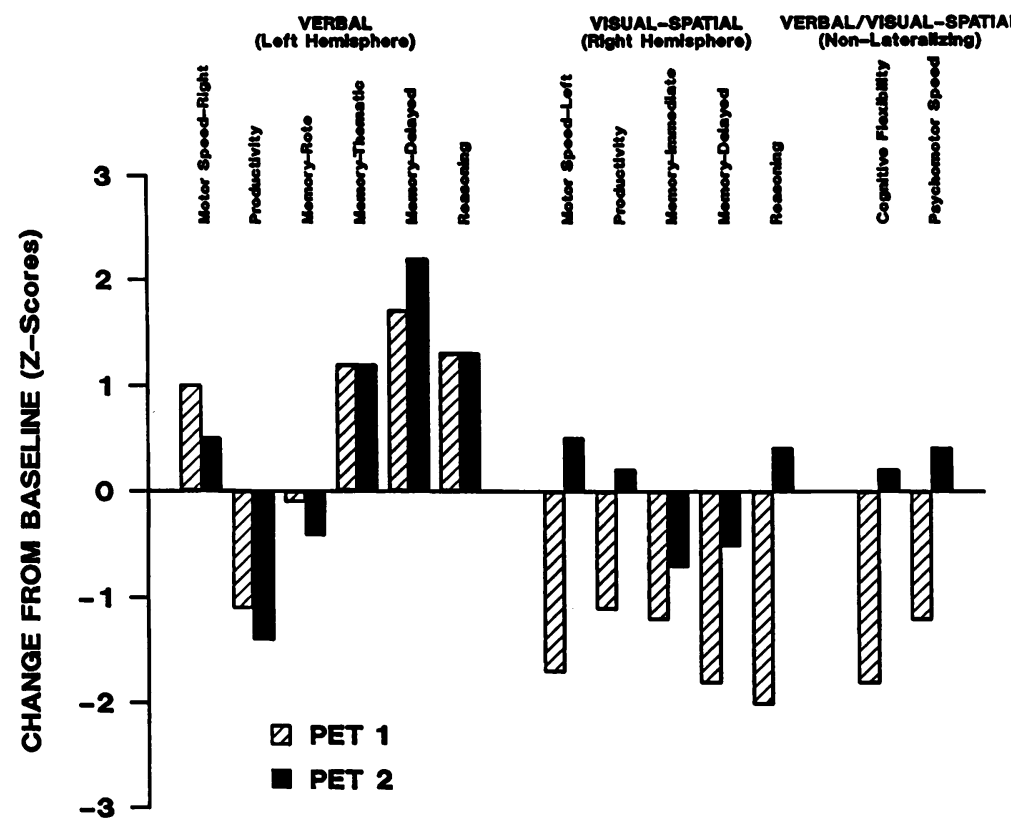

Figure 2 Changes in performance from baseline (Case 1) on summary variables, grouped according to whether primarily verbal (left hemisphere) or visual-spatial (right hemisphere) processes are involved. The baseline was obtained two years before the first PET scan. A change of -0.6 to -1.3 (z-score) units indicates a mild-to-moderate deterioration of performance, -1.3 to -2.0 a moderate-to-marked change, and more than two units a severe change. ${ }^{23}$ This patient showed a generalised deterioration in visual-spatial functions at the time of PET l; eight months post infarct, her functioning had returned to baseline (unimpaired) levels.

\section{CASE 2 COGNITIVE VARIABLES}

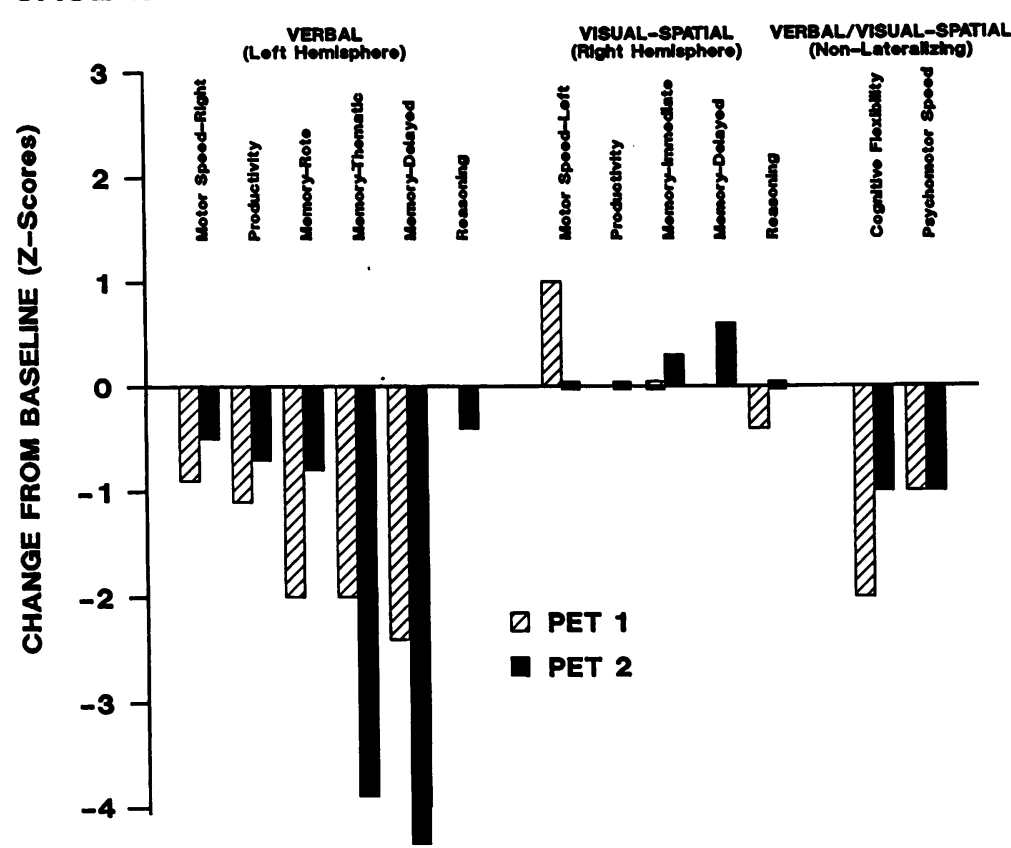

Figure 3 Changes from baseline performance (Case 2). At the time of the first PET scan, she showed little or no change in visual-spatial (right hemisphere) functions, but widespread changes on verbal and non-lateralising summary variables. These deficits were still present ten months later, although her speech and motor functioning had improved.

Case 2 The first PET scan (fig lb) showed a reduction in glucose metabolism in the left superior parietal and posterior temporal regions. Less marked but similarly distributed abnormalities were present in the right hemisphere. The second scan showed the same distribution of abnormalities on both sides, but these were now more severe. In addition, there was a new loss of glucose metabolism in the left pericentral region.

This patient showed fairly diffuse cognitive dysfunction on initial testing one year before her CVA although she did not have clinically active CNS involvement. She had particular difficulty with visual-spatial reasoning which was compatible with either left- or right-sided parietal dysfunction. A second testing one month before her CVA had been done to evaluate changes after several months of increased prednisone dosage. Her performance on verbal productivity and memory tasks was generally better than on initial testing.

At the time of the first PET scan two months post-infarct, she showed appreciable deterioration from her baseline performance three months earlier in cognitive flexibility and verbal memory, and a mild to moderate decline in verbal productivity and right hand finger tapping rate (fig 3 ). These findings were consistent with a recent left hemisphere lesion. When tested ten months later, her memory and visual-spatial deficits had not resolved; the significant losses in memory function from the levels immediately before the CVA were still evident as were the changes in non-specific indicators of CNS dysfunction, that is, cognitive flexibility and psychomotor speed.

Case 3 The first PET scan (fig lc) showed reduction of cortical glucose metabolism that involved the whole of the pericentral region on the left and most of the left parietal lobe. There was also some reduction of glucose consumption in the posterior part of the right parietal lobe. The second PET scan showed a return towards a more uniform distribution of cortical glucose consumption in both hemispheres, especially on the left. However, associated with this pericentral and parietal improvement, there was a concomitant fall in frontal glucose consumption. This was not seen in the other two patients.

This patient showed fairly widespread cognitive deficits at the time of the first PET scan, with extremely slow responses suggesting involvement of subcortical structures. She was significantly impaired for verbal and visualspatial productivity, visual-spatial reasoning and memory, cognitive flexibility and psychomotor speed. Relative to this initial baseline testing, her performnce at the time of the second PET study was significantly poorer in several areas, suggesting increased dysfunction primarily in the anterior regions (fig 4). Cognitive flexibility was considerably reduced, and she showed a slower finger tapping rate on both hands, poorer verbal productivity and poorer immediate memory for thematic verbal material. No changes were indicated in visualspatial functioning.

\section{Discussion}

The first PET scan of each of the patients we describe showed regional abnormalities of glucose metabolism which corresponded to the 


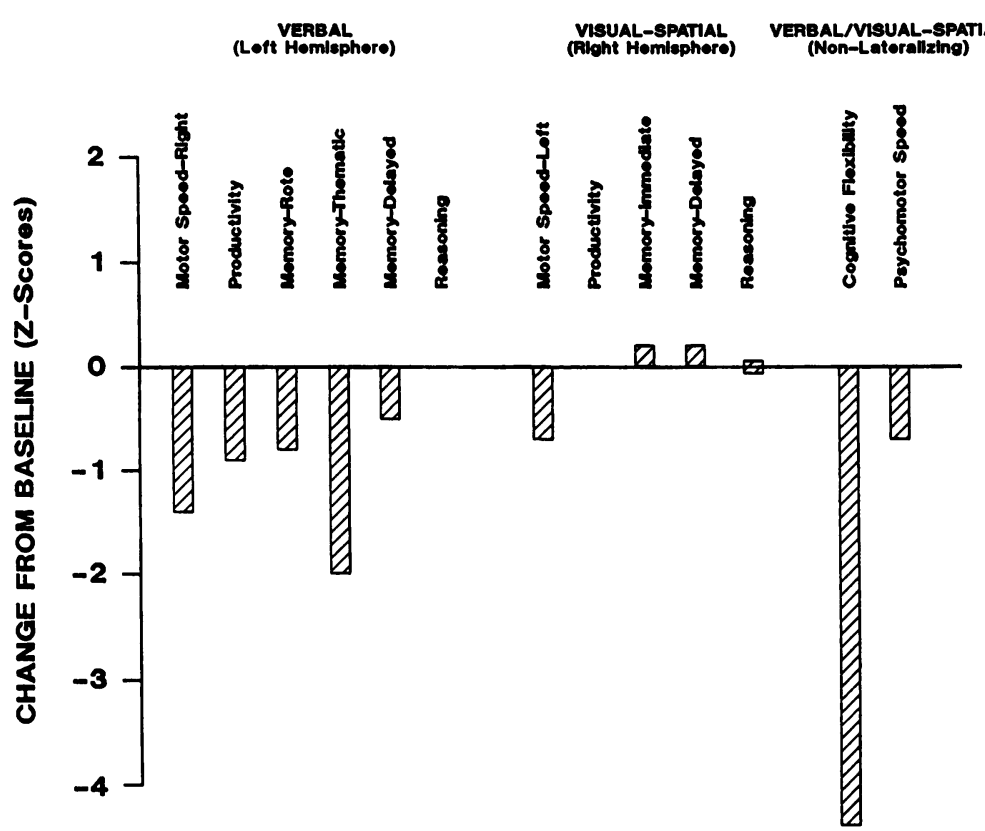

Figure 4 Changes from baseline performance (case 3), at the time of her second PET scan after eight months of increased and then tapered steroid treatment. At the time of the first scan (baseline), she had shown marked visual-spatial deficits and speech dysfluency, but relatively intact rote verbal memory. structural lesions seen on the CT scan. In addition, PET showed appreciable further functional abnormalities. Although case 1 had mild neurological deficits, her neurocognitive profile indicated widespread right hemisphere dysfunction. This was paralleled by reductions in glucose metabolism in the right hemisphere, suggesting far more extensive CNS dysfunction than would have been predicted on the basis of the fairly small, discrete lesion in the basal ganglia seen on CT scan. Wide-spread suppression of glucose metabolism soon after stroke has been documented previously, ${ }^{28}$ as well as some resolution of abnormalities together with the resolution of associated cognitive deficits as we found in this patient. In case 2, the CT scan indicated a unilateral (left) parietal infarct, but PET showed a bilateral reduction in glucose metabolism in the temporal regions as well. As in case 1 , the PET abnormalities suggested far more extensive CNS dysfunction than the CT scan indicated. In contrast to case 1 , however, the abnormalities in glucose metabolism did not resolve over time; they got worse. In parallel with the PET findings, cognitive performance deteriorated.

PET identified extensive significant functional alterations in these SLE patients. The pathogenesis of this brain dysfunction may include thrombosis associated with lupus anticoagulant, present in all three cases, and/or neuronal antibodies ${ }^{29}$ directed against specific; new antigens ${ }^{22}$ which were present in case 2 . Although CT scan identified circumscribed brain lesions, the more extensive functional alterations shown by PET may have structural correlates not detectable by CT scanning, which is much less sensitive to structural lesions in NP-SLE than MRI. ${ }^{13}$ The regional alterations in glucose metabolism in cases 1 and 2 corresponded well to the functional deficits identified by cognitive testing, and the findings on repeated scanning were paralleled by findings from neurocognitive testing. This provides further support for our suggestion that the high prevalence of cognitive impairment in SLE patients ${ }^{7}$ reflects primary CNS involvement, even in those patients without clinical evidence of active CNS involvement.

Although the findings from PET and neurocognitive testing in case 3 appear to correspond less well than in Cases 1 and 2, the discrepancies stimulate interesting hypotheses. At the time of the second PET scan, performance on tests of frontal functioning was poorer than it had been initially; these performance changes did correspond to the reductions in frontal glucose consumption seen in the second PET scan. However, there was also some resolution of the original PET abnormalities in the posterior regions, which was not accompanied by an improvement in performance. One possible reason for this discrepancy is that the deterioration of frontal functioning had more significant effects on performance than did the improvement in parietal-temporal functioning, particularly if the task required planning, self-monitoring or hypothesis generation which are well established as functions sensitive to integrity of the frontal regions. ${ }^{30}$ Moreover, frontal dysfunction has been reported to lead to impairment in the acquisition and retention of information, ${ }^{30}$ seen particularly as an exaggerated inability to maintain new information in the face of interference. PET and blood flow studies of normal individuals are providing converging evidence of a significant role of the frontal regions in memory tasks. ${ }^{31}$ Finally, it is possible that even though an increase in glucose consumption was indicated on the second scan, the parietaltemporal areas may still not have had a normal distribution of glucose consumption (for example, compared with the second scan of the left hemisphere of case 1 ). The lack of improvement in performance, despite some normalisation of the distribution of glucose consumption in this patient points to the need for complementing physiological studies of brain function with behavioural indices, so as to establish the significance of physiological alterations.

In case 3 , the reduction of the prednisone dose was the only intervention in the interval between the two scans, and no significant clinical changes were noted. The issue of the effects of steroids on CNS status is controversial. There are anecdotal reports of both steroid-induced $^{32}$ and steroid-sensitive ${ }^{3334}$ dementia as well as steroid-induced psychosis. ${ }^{35}$ Our cross-sectional data have suggested no negative effects of steroids on cognitive status $^{78}$ while our longitudinal data have suggested improvement with an initiation or increase of steroid dosage. ${ }^{3637}$ There has been a recent report of a normalisation of the distribution of glucose metabolism (paralleled 
by an improvement in cognitive performance) in patients treated with zidovudine for dementia associated with acquired immunodeficiency syndrome. ${ }^{38}$ This is further evidence that cerebral metabolism fluctuates and can be affected by certain therapeutic interventions.

In summary, functional indices of cerebral integrity such as glucose metabolism and neuropsychological performance are proving to be much more sensitive ways of documenting "subclinical" CNS involvement in NP-SLE than structural indices. We are currently examining the PET correlates of specific cognitive deficits in SLE patients without identified CNS lesions. In addition, PET and neurocognitive testing may prove to be very useful in monitoring the response to therapeutic intervention. While PET and/or MRI can yield critical information as to the presence of CNS abnormalities, only neuropsychological testing can document and monitor the often fairly extensive behavioural sequelae of discrete brain lesions.

This work was supported by grants from The Lupus Society of Hamilton, The Arthritis Society of Canada, the St. Joseph's Hospital Foundation, the Ontario Mental Health Foundation and the Medical Research Council of Canada. A preliminary version of these results was presented at the 52nd annual meeting of the American Rheumatism Association in Houston Texas (23-28 May 1988).

We thank Professor John Kushner who made ${ }^{18} \mathrm{~F}$ available to us from the McMaster van der Graaf Accelerator and Julie Schmidt who did the neurocognitive testing of these patients.

1 Kassan SS, Lockshin MD. Central nervous system lupus erythematosus. Arthritis Rheum 1979;22:1382-5.

2 McCune WJ, Golbus J. Neuropsychiatric lupus. Rheum Dis Clin N Am 1988;14:149-67.

3 Zvaifler NJ, Bluestein HG. The pathogenesis of central nervous system manifestations of systemic lupus erythematosus. Arthritis Rheum 1982;25:862-6.

4 Bresnihan B. CNS lupus. Clin Rheum Dis 1982;8:183-5.

5 Zvaifler NJ. Neurological manifestations. In: Schur PH, ed. The clinical management of systemic lupus erythematosus. The clinical management of systemic lupus eryth

6 Denburg JA, Temesvari P. The pathogenesis of neuropsychiatric lupus. Can Med Assoc $\mathcal{F}$ 1983;128:257-60.

7 Carbotte RM, Denburg SD, Denburg JA. Prevalence of cognitive impairment in systemic lupus erythematosus. $\mathcal{F}$ Nerv Ment Dis 1986;174:357-64.

8 Denburg SD, Carbotte RM, Denburg JA. Cognitive impairment in systemic lupus erythematosus: A neuropsychological study of individual and group deficits. $\mathcal{f}$ Clin Exp Neuropsychol 1987;9:323-39.

9 Carbotte RM, Denburg SD, Long AA, Singer J, Denburg JA. The contribution of disease activity to cognitive deficit in systemic lupus erythematosus (SLE). $\boldsymbol{f}$ Clin Exp Neuropsychol 1987;9:64-5.

10 Levin HS, Amparo EG, Eisenberg HM et al. Magnetic resonance imaging and computerized tomography in relation to the neurobehavioral sequelae of mild and relation to the neurobehavioral sequelae of mild and
moderate head injuries. $₹$ Neurosurg 1987;66:706-13.

moderate head injuries. F Neurosurg 1987;66:706-13.
11 Hart RP, Kreutzer JS. Renal system. In: Tarter RE, Van Thiel DH, Edwards KL, eds. Medical neuropsychology; the impact of disease on behavior. New York: Plenum Press, 1988:99-120.

12 Gibson T, Myers AR. Nervous system involvement in systemic lupus erythematosus. Ann Rheum Dis 1976;35:398-406.

13 McCune WJ, MacGuire A, Aisen A, Gebarski S. Identification of brain lesions in neuropsychiatric systemic lupus erythematosus by magnetic resonance scanning. Arthritis Rheum 1988;31:159-66.

14 Sibbitt WL, Sibbitt RR, Griffey RH, Eckel C, Bankhurst $\mathrm{AD}$. Magnetic resonance imaging in the evaluation of acute neuropsychiatric disease in systemic lupus erythematosus. Ann Rheum Dis 1989;48:1014-22.

15 Hiraiwa $M$, Nonaka $C$, Abe $T$, lio $M$. Positron emission tomography in systemic lupus erythematosus: relation of cerebral vasculitis to PET findings. Am $\mathfrak{f}$ Neuroradiol 1983;4:541-3.

16 Volkow ND, Warner N, McIntyre R, Valentine A. Cerebral involvement in systemic lupus erythematosus. $A m \mathcal{J}$ Physiol Imaging 1988;3:91-8.

17 Stoppe G, Wildhagen K, Seidel JW, et al. Positron emission tomography in neuropsychiatric lupus erythematosus. Neurology 1990;40:304-8.

18 Pinching AJ, Travers RL, Hughes GRV, Jones T, Moss S Oxygen-15 brain scanning for detection of cerebral involvement in systemic lupus erythematosus. Lance 1978;1:898-900.

19 Awada HH, Mamo HL, Luft AG, Ponsin JC, Kahn MF. Cerebral blood flow in systemic lupus with and without central nervous system involvement. $\mathcal{f}$ Neurol Neurosurg Pychiatry 1987;50:1597-1601.

20 Kushner MJ, Chawluk MD, Fazekas F, et al. Cerebral blood flow in systemic lupus erythematosus with or without flow in systemic lupus erythematosus with or with

21 The ARA Subcommittee for SLE Criteria. The 1982 revised criteria for the classification of systemic lupus erythematosus. Arthritis Rheum 1982;25:1271-7.

22 Hanly JG, Rajaraman S, Behmann S, Denburg JA. A novel neuronal antigen identified by sera from patients with systemic lupus erythematosus. Arthritis Rheum 1988;31:1492-9.

23 Lezak MD. Neuropsychological assessment, 2nd ed. New York: Oxford University Press 1983.

24 Nahmias C. The McMaster positron emission tomograph: Design and evaluation. Nuclear Instrument Method 1984,1:113-4.

25 Brownell GL, Kearfolt KJ, Kairento AL, et al. Quantitation of regional cerebral glucose metabolism. $\mathcal{f}$ Comput Assist of regional cerebral gluco

26 Nahmias C, Loken $M$, Garnett ES. Display of local metabolic rates from the human brain. Proc 6th Aachen Symposium on signal theory, multi-dimensional signals and image processing. Heidelberg: Springer Verlag, 1987.

27 Friston KJ, Passingham RE, Nutt JG, et al. Localization in PET images: Direct fitting of the intercommissural (AcPc) line. $\mathcal{f}$ Cereb Blood Flow Metab 1989;9:690-5.

28 Kuhl DE, Phelps ME, Kowell AP, Metter EJ, Selin C, Winter J. The effects of stroke on local cerebral metabolism and perfusion. Mapping by emission computed tomography of 18FDG and 13NH3. Ann Neurol 1980;8:47-60.

29 Denburg JA, Carbotte RM, Denburg SD. Neuronal antibodies and cognitive function in systemic lupus erythemabodies and cognitive function in syst
tosus. Neurology 1987;37:464-7.

30 Stuss DT, Benson DF. The frontal lobes. New York: Raven Press, 1986.

31 Duara R, Loewenstein DA, Barker WW. Utilization of behavioral activation paradigms for positron emission tomography studies in normal young and elderly subjects and in dementia. In: Duara R, ed. Positron emission tomography in dementia. New York: Wiley-Liss, 1990:131-48.

32 Varney NR, Alexander B, MacIndoe JH. Reversible steroid dementia in patients without steroid psychosis. $A m \mathcal{F}$ Psychiatry 1984;141:369-72.

33 Paulson GW. Steroid-sensitive dementia. Am f Psychiatry 1983;140:1031-3.

34 Chynoweth $R$, Foley J. Pre-senile dementia responding to steroid therapy. $B r \mathcal{F}$ Psychiatry 1969;115:703-8.

35 Ling MHM, Perry PJ, Tsuang MT. Side effects of corticosteroid therapy: Psychiatric aspects. Arch Gen Psychiatry 1981;38:471-7.

36 Denburg SD, Carbotte RM, Denburg JA. The effects of corticosteroids on neurocognitive functioning: A trend toward improvement. Canadian Psychol 1987;28:132.

37 Denburg SD, Carbotte RM, Guyatt GD, Denburg JA Effects of corticosteroids on neuropsychological functioning: Results of single patient $(\mathrm{N}$ of 1$)$ trials. $\mathcal{f}$ Clin Exp Neuropsychol 1989;11:65.

38 Cohen YAR, Brouwer P, Spitzer A, et al. Response of humanimmunodeficiency-virus-associated neurological disease to 3-azido-3-deoxythymidine. Lancet 1987;1: 132-5. 